\title{
Cluster formation and its role in the elimination of azeotrope of the acetone-methanol mixture by ionic liquid
}

Fang Chen, Long Zhang, Zhiping Liu* and Guangren Yu* ${ }^{a}$ State Key Laboratory of Organic-Inorganic Composites, Beijing Advanced Innovation Center for Soft Matter Science and Engineering, Beijing University of Chemical Technology, Beijing 100029, P. R. China 


\section{Supporting Information}

Table S1. Force field parameters

\begin{tabular}{|c|c|c|c|}
\hline \multicolumn{4}{|c|}{ Non-bonded parameters } \\
\hline Atom & $\begin{array}{l}\sigma(\AA) \\
\quad \text { acetone }\end{array}$ & $\varepsilon\left(\mathrm{kJ} * \mathrm{~mol}^{-1}\right)$ & q (e) \\
\hline $\mathrm{CH} 3$ & 3.75 & 0.81588 & -0.049 \\
\hline $\mathrm{O}$ & 3.05 & 0.656888 & -0.564 \\
\hline $\mathrm{C}$ & $\begin{array}{l}3.82 \\
\text { methanol }\end{array}$ & 0.225936 & 0.662 \\
\hline $\mathrm{CH} 3$ & 3.75 & 0.81588 & 0.265 \\
\hline $\mathrm{O}$ & 3.02 & 0.77404 & -0.7 \\
\hline $\mathrm{H}$ & $\begin{array}{l}0 \\
{\left[\text { MMIM }^{+}\right.}\end{array}$ & 0 & 0.435 \\
\hline $\mathrm{N}$ & 3.25 & 0.71128 & 0.01875 \\
\hline $\mathrm{CR}$ & 3.4 & 0.359824 & 0 \\
\hline $\mathrm{CW}$ & 3.4 & 0.359824 & -0.2 \\
\hline $\mathrm{CH} 3$ & 3.66 & 0.79496 & 0.3375 \\
\hline H5 & 1.247 & 0.12552 & 0.1875 \\
\hline $\mathrm{H} 4$ & $\begin{array}{l}1.604 \\
{\left[\text { [DMP] }^{-}\right.}\end{array}$ & 0.12552 & 0.25 \\
\hline $\mathrm{P}$ & 3.74 & 0.8786 & 1.0848 \\
\hline $\mathrm{O} 2$ & 2.96 & 0.8368 & -0.7346 \\
\hline OS & 3 & 0.7113 & -0.4494 \\
\hline $\mathrm{CH} 3$ & 3.4 & 0.5021 & 0.1416 \\
\hline \multicolumn{4}{|c|}{ Bonded parameters } \\
\hline Bonds & $\begin{array}{c}\mathrm{Kr}\left(\mathrm{kJ} * \mathrm{~mol}^{-1} * \AA^{-2}\right) \\
\text { acetone }\end{array}$ & r0 (Å) & \\
\hline $\mathrm{C}-\mathrm{CH} 3$ & 1326.328 & 1.52 & \\
\hline $\mathrm{C}=\mathrm{O}$ & $\begin{array}{r}2384.88 \\
\text { methanol }\end{array}$ & 1.229 & \\
\hline $\mathrm{CH} 3-\mathrm{O}$ & 1338.88 & 1.43 & \\
\hline $\mathrm{O}-\mathrm{H}$ & $\begin{array}{l}2313.752 \\
\text { [MMIM] }^{+}\end{array}$ & 0.945 & \\
\hline $\mathrm{N}-\mathrm{CR}$ & 1719.624 & 1.325 & \\
\hline $\mathrm{N}-\mathrm{CW}$ & 1719.624 & 1.378 & \\
\hline CW-CW & 1999.952 & 1.343 & \\
\hline $\mathrm{N}-\mathrm{CH} 3$ & 1079.472 & 1.5 & \\
\hline CR-H5 & 1439.296 & 1.07 & \\
\hline CW-H4 & $\begin{array}{l}1439.296 \\
\text { [DMP }^{-}\end{array}$ & 1.07 & \\
\hline $\mathrm{P}-\mathrm{O} 2$ & 2196.6 & 1.48 & \\
\hline
\end{tabular}




\begin{tabular}{|c|c|c|c|}
\hline P-OS & 962.32 & 1.61 & \\
\hline OS-CH3 & 1338.88 & 1.41 & \\
\hline Angles & $\mathrm{K \theta}\left(\mathrm{kJ}^{*} \mathrm{~mol}^{-1 *} \mathrm{rad}^{-2}\right)$ & $\theta 0\left({ }^{\circ}\right)$ & \\
\hline \multicolumn{4}{|c|}{ acetone } \\
\hline $\mathrm{CH} 3-\mathrm{C}=\mathrm{O}$ & 519.611 & 121.4 & \\
\hline $\mathrm{CH} 3-\mathrm{C}-\mathrm{CH} 3$ & 519.611 & 117.2 & \\
\hline \multicolumn{4}{|c|}{ methanol } \\
\hline $\mathrm{CH} 3-\mathrm{O}-\mathrm{H}$ & 460.5747 & 108.5 & \\
\hline \multicolumn{4}{|c|}{ [MMIM] $^{+}$} \\
\hline CW-N-CR & 283.6752 & 108 & \\
\hline N-CR-N & 308.3608 & 109.9 & \\
\hline $\mathrm{N}-\mathrm{CW}-\mathrm{CW}$ & 292.0432 & 107.1 & \\
\hline N-CR-H5 & 214.2208 & 125.7 & \\
\hline CR-N-CH3 & 268.6128 & 126.3 & \\
\hline CH3-N-CW & 268.6128 & 125.7 & \\
\hline $\mathrm{N}-\mathrm{CW}-\mathrm{H} 4$ & 214.2208 & 122.1 & \\
\hline $\mathrm{H} 4-\mathrm{CW}-\mathrm{CW}$ & 209.2 & 130.7 & \\
\hline \multicolumn{4}{|c|}{$[\mathbf{D M P}]^{-}$} \\
\hline $\mathrm{O} 2-\mathrm{P}-\mathrm{O} 2$ & 585.76 & 119.9 & \\
\hline O2-P-OS & 418.4 & 108.23 & \\
\hline OS-P-OS & 188.28 & 102.6 & \\
\hline CH3-OS-P & 418.4 & 120.5 & \\
\hline Dihedrals & $\mathbf{n}$ & $\mathbf{K} \chi\left(\mathbf{k J} * \mathrm{~mol}^{-1}\right)$ & $\delta($ degree $)$ \\
\hline \multicolumn{4}{|c|}{ [MMIM] $^{+}$} \\
\hline H4-CW-N-CH3 & 2 & 6.276 & 180 \\
\hline $\mathrm{H} 4-\mathrm{CW}-\mathrm{N}-\mathrm{CR}$ & 2 & 8.368 & 180 \\
\hline CW-CW-N-CR & 2 & 50.208 & 180 \\
\hline N-CW-CW-H4 & 2 & 6.276 & 180 \\
\hline $\mathrm{N}-\mathrm{CW}-\mathrm{CW}-\mathrm{N}$ & 2 & 50.208 & 180 \\
\hline CH3-N-CR-N & 2 & 8.368 & 180 \\
\hline CH3-N-CR-H5 & 2 & 6.276 & 180 \\
\hline CW-N-CR-N & 2 & 50.208 & 180 \\
\hline CW-N-CR-H1 & 2 & 6.276 & 180 \\
\hline CH3-N-CW-CW & 2 & 8.368 & 180 \\
\hline $\mathrm{H} 2-\mathrm{CW}-\mathrm{CW}-\mathrm{H} 2$ & 2 & 6.276 & 180 \\
\hline \multicolumn{4}{|c|}{$[\mathbf{D M P}]^{-}$} \\
\hline $\mathrm{CH} 3-\mathrm{OS}-\mathrm{P}-\mathrm{O} 2$ & 1 & 5.021 & 0 \\
\hline CH3-OS-P-OS & 1 & 1.046 & 0 \\
\hline Improper torsions & $\mathbf{n}$ & $\mathbf{K} \chi\left(\mathbf{k J}^{*} \mathrm{~mol}^{-1}\right)$ & $\delta($ degree $)$ \\
\hline \multicolumn{4}{|c|}{ [MMIM] $^{+}$} \\
\hline N-N-CR-H1 & 2 & 4.6024 & 180 \\
\hline CR-CW-N-CH3 & 2 & 8.368 & 180 \\
\hline N-CW-CW-H2 & 2 & 4.6024 & 180 \\
\hline
\end{tabular}


Table S2. Optimized structure and binding energies ${ }^{\mathrm{a}}\left(\mathrm{E}_{\mathrm{b}}, \mathrm{kJ} / \mathrm{mol}\right)$ of some dimers by QM (Quantum Mechanics) and MM (Molecular Mechanics)

\begin{tabular}{|c|c|c|c|c|c|c|c|}
\hline \multirow{2}{*}{\multicolumn{2}{|c|}{ Molecule A }} & \multirow{2}{*}{ Molecule B } & \multirow{2}{*}{ Hydrogen bond } & \multicolumn{2}{|c|}{ 1_O...H $(\AA)$} & \multicolumn{2}{|c|}{$\mathrm{E}_{\mathrm{b}}$} \\
\hline & & & & $\mathrm{QM}^{\mathrm{b}}$ & $\mathrm{MM}^{\mathrm{c}}$ & QM & MM \\
\hline & methanol & {$[\mathrm{MMIM}]^{+}$} & O...H5-CR & 2.03 & 1.95 & -59.3 & -53.2 \\
\hline & methanol & {$[\mathrm{DMP}]^{-}$} & $\mathrm{O}-\mathrm{H} \ldots \mathrm{O} 2$ & 1.73 & 1.61 & -83.1 & -69.2 \\
\hline & methanol & {$[\mathrm{DMP}]^{-}$} & O-H...OS & 1.82 & 1.81 & -69.0 & -49.2 \\
\hline & methanol & methanol & $\mathrm{O}-\mathrm{H} \ldots \mathrm{O}$ & 1.88 & 1.88 & -61.7 & -56.4 \\
\hline & acetone & {$[\mathrm{MMIM}]^{+}$} & $\mathrm{O} \ldots \mathrm{H} 5-\mathrm{CR}$ & 2.05 & 1.97 & -70.4 & -54.9 \\
\hline & acetone & {$[\mathrm{DMP}]^{-}$} & & & & -71.4 & -46.7 \\
\hline a) & \multicolumn{7}{|c|}{$\begin{array}{l}\text { The binding energy } \mathrm{E}_{\mathrm{b}} \text { of molecule } \mathrm{A} \text { and } \mathrm{B} \text { was calculated as } E_{b}=E_{(A+B)}-E_{A}-E_{B} \\
\text { where } \mathrm{E}_{(\mathrm{A}+\mathrm{B})} \text { refers to energy of dimer of molecule } \mathrm{A} \text { and } \mathrm{B} \text {, and } \mathrm{E}_{\mathrm{A}}\left(\mathrm{E}_{\mathrm{B}}\right) \text { is the energy of } \\
\text { single molecule } \mathrm{A}(\mathrm{B}) \text { with the same structure in the dimer. }\end{array}$} \\
\hline b) & \multicolumn{7}{|c|}{$\begin{array}{l}\text { Calculated by Gaussian } 09 \text {, revision D. } 01^{(1)} \text {, with the density functional theory of B3LYP- } \\
\text { D3 }{ }^{(2)-(6)} \text { employed with the } 6-31+\mathrm{G}^{* *} \text { basis sets }{ }^{(7),(8)} \text {. }\end{array}$} \\
\hline c) & Calculated $\mathrm{t}$ & program molec & lar modeling packa & . & & & \\
\hline
\end{tabular}


Table S3. Error estimates on interaction energy ( $\mathrm{kcal} / \mathrm{mol})$ in simulation for the system of $x_{\text {acetone }}=0.95^{a}$

\begin{tabular}{ccc}
\hline interaction energy & average & standard deviation \\
\hline pairwise energy & -19800.00 & 2.97 \\
vdw $^{\mathrm{b}}$ & -8526.55 & 2.41 \\
elec $^{\mathrm{c}}$ & -11931.20 & 2.73 \\
tail $^{\mathrm{d}}$ & -381.25 & 0.07 \\
total potential energy $_{\text {kinetic energy }}$ & -16360.60 & 2.78 \\
$\mathrm{~A}-\mathrm{M}^{\mathrm{e}}$ & 96645.77 & 0.65 \\
$\mathrm{M}-\mathrm{M}$ & -323.612 & 4.17 \\
[MMIM] $^{+}-\mathrm{M}$ & -11.18 & 0.66 \\
[DMP] $^{-} \mathrm{M}$ & -386.93 & 6.43 \\
$\mathrm{~A}^{\mathrm{f}}$ & -791.36 & 6.75 \\
$\mathrm{M}^{\mathrm{g}}$ & -6.57 & 0.003 \\
\hline
\end{tabular}

a) The total molecules number of acetone and methanol is 1600 in this system. 80 ion pairs were added to the mixture. We extracted energy data in every step from 20 ns simulation. Therefore, totally 10 million data points were used for every energy term,

b) van der Waals pairwise energy

c) Coulombic pairwise energy + long-range kspace energy

d) van der Waals energy long-range tail correction

e) X-M refers to the interaction energy between $\mathrm{X}$ and $\mathrm{M}$. A: acetone; M: methanol

f) interaction energies belonged to acetone, by per mole of acetone

g) interaction energies belonged to methanol, by per mole of methanol 
Table S4. Breakdown of interaction energies for methanol and acetone $(\mathrm{kJ} / \mathrm{mol}){ }^{\text {a }}$

\begin{tabular}{|c|c|c|c|c|c|c|c|c|c|c|}
\hline \multicolumn{11}{|c|}{ systems without [MMIM][DMP] } \\
\hline & \multicolumn{5}{|c|}{ methanol } & \multicolumn{5}{|c|}{ acetone } \\
\hline $\mathrm{x}_{1}$ & \multicolumn{2}{|c|}{$M-A^{b}$} & M-M & \multicolumn{2}{|c|}{ sum } & \multicolumn{2}{|c|}{ A-A } & A-M & \multicolumn{2}{|c|}{ sum } \\
\hline 0 & \multicolumn{2}{|c|}{0} & -39.19 & \multicolumn{2}{|c|}{-39.19} & & & & \\
\hline 0.05 & \multicolumn{2}{|c|}{-1.57} & -37.29 & \multicolumn{2}{|c|}{-38.86} & \multicolumn{2}{|c|}{-2.32} & -29.80 & \multicolumn{2}{|c|}{-32.11} \\
\hline 0.23 & \multicolumn{2}{|c|}{-6.40} & -30.95 & \multicolumn{2}{|c|}{-37.35} & \multicolumn{2}{|c|}{-9.42} & -21.43 & \multicolumn{2}{|c|}{-30.85} \\
\hline 0.41 & \multicolumn{2}{|c|}{-10.63} & -25.45 & \multicolumn{2}{|c|}{-36.08} & \multicolumn{2}{|c|}{-15.12} & -15.30 & \multicolumn{2}{|c|}{-30.42} \\
\hline 0.59 & \multicolumn{2}{|c|}{-14.61} & -19.71 & \multicolumn{2}{|c|}{-34.32} & \multicolumn{2}{|c|}{-19.64} & -10.15 & \multicolumn{2}{|c|}{-29.79} \\
\hline 0.775 & \multicolumn{2}{|c|}{-19.01} & -12.65 & \multicolumn{2}{|c|}{-31.66} & \multicolumn{2}{|c|}{-23.49} & -5.52 & \multicolumn{2}{|c|}{-29.01} \\
\hline 0.9 & \multicolumn{2}{|c|}{-22.55} & -6.48 & -29 & & -25.8 & & -2.51 & \multicolumn{2}{|c|}{-28.40} \\
\hline 0.95 & -24 & & -3.59 & -27 & & -26.7 & & -1.27 & & \\
\hline 1 & & & & & & -27.7 & & 0 & & \\
\hline & & & & systems & ith [M] & $\mathrm{IM}][\mathrm{DM}$ & & & & \\
\hline & & & thanol & & & & & acetone & & \\
\hline $\mathrm{x}_{1}{ }^{\prime}$ & M-A & M-M & $\begin{array}{c}\text { M- } \\
{\left[\mathrm{MMIM}^{+}\right.}\end{array}$ & $\begin{array}{c}\text { M- } \\
{\left[\mathrm{DMP}^{-}\right.}\end{array}$ & sum & A-M & A-A & $\begin{array}{c}\text { A- } \\
{[\mathrm{MMIM}]^{+}}\end{array}$ & $\begin{array}{c}\text { A- } \\
{[\mathrm{DMP}]^{-}}\end{array}$ & sum \\
\hline 0 & 0 & -28.73 & -3.54 & -7.85 & -40.12 & & & & & \\
\hline 0.05 & -1.22 & -27.11 & -3.52 & -8.01 & -39.86 & -23.20 & -2.19 & -5.91 & -1.37 & -32.67 \\
\hline 0.23 & -4.92 & -21.98 & -3.37 & -8.57 & -38.84 & -16.48 & -8.96 & -5.11 & -1.23 & -31.78 \\
\hline 0.41 & -7.87 & -16.86 & -3.45 & -9.70 & -37.89 & -11.33 & -14.40 & -4.22 & -1.02 & -30.97 \\
\hline 0.59 & -10.05 & -11.61 & -3.86 & -11.54 & -37.06 & -6.98 & -18.77 & -3.57 & -0.86 & -30.19 \\
\hline 0.775 & -11.30 & -5.78 & -5.19 & -14.45 & -36.72 & -3.28 & -22.70 & -2.96 & -0.42 & -29.35 \\
\hline 0.9 & -10.04 & -1.63 & -7.22 & -19.44 & -38.33 & -1.12 & -24.88 & -2.47 & -0.30 & -28.77 \\
\hline 0.95 & -8.46 & -0.58 & -10.12 & -20.73 & -39.90 & -0.45 & -25.81 & -2.28 & -0.01 & -28.56 \\
\hline 1 & & & & & & 0 & -26.37 & -2.10 & -0.07 & -28.54 \\
\hline
\end{tabular}

a) The breakdown of interaction energies on methanol and acetone were calculated by per mole of methanol and acetone, e.g., for interaction M-A the breakdown of interaction energies on methanol $\left(\mathrm{E}^{\text {per } \mathrm{M}}{ }_{\mathrm{M}-\mathrm{A}}\right)$ and acetone $\left(\mathrm{E}^{\text {per } \mathrm{A}}{ }_{\mathrm{M}-\mathrm{A}}\right)$ were calculated as:

$$
\begin{gathered}
E^{\text {per }{ }_{M-A}}=E^{\text {sys }}{ }_{M-A} / n_{M} \\
E^{\text {per }{ }_{M-A}}=E^{\text {sys }}{ }_{M-A} / n_{A}
\end{gathered}
$$

where $\mathrm{E}_{\mathrm{M} \text {-A }}^{\text {sys }}$ refers to the interaction energy for M-A in the whole system.

b) $\mathrm{M}$ refers to methanol, A refers to acetone. 


\section{References}

(1) Frisch, M. J.; Trucks, G. W.; Schlegel, H. B.; Scuseria, G. E.; Robb, M. A.; Cheeseman, J. R.; Scalmani, G.; Barone, V.; Mennucci, B.; Petersson, G. A.; Nakatsuji, H.; Caricato, M.; Li, X.; Hratchian, H. P.; Izmaylov, A. F.; Bloino, J.; Zheng, G.; Sonnenberg, J. L.; Hada, M.; Ehara, M.; Toyota, K.; Fukuda, R.; Hasegawa, J.; Ishida, M.; Nakajima, T.; Honda, Y.; Kitao, O.; Nakai, H.; Vreven, T. ; Montgomery, J. A., Jr.; Peralta, J. E.; Ogliaro, F.; Bearpark, M.; Heyd, J. J.; Brothers, E.; Kudin, K. N.; Staroverov, V. N.; Keith, T.; Kobayashi, R.; Normand, J.; Raghavachari, K.; Rendell, A.; Burant, J. C.; Iyengar, S. S.; Tomasi, J.; Cossi, M.; Rega, N.; Millam, J. M.; Klene, M.; Knox, J. E.; Cross, J. B.; Bakken, V.; Adamo, C.; Jaramillo, J.; Gomperts, R.; Stratmann, R. E.; Yazyev, O.; Austin, A. J.; Cammi, R.; Pomelli, C.; Ochterski, J. W.; Martin, R. L.; Morokuma, K.; Zakrzewski, V. G.; Voth, G. A.; Salvador, P.; Dannenberg, J. J.; Dapprich, S.; Daniels, A. D.; Farkas, O.; Foresman, J. B.; Ortiz, J. V.; Cioslowski, J.; Fox, D. J. Gaussian, Inc., Wallingford $\mathrm{CT}, \mathbf{2 0 1 3}$

(2) Lee, C.; Yang, W.; Parr, R. G. Development of the Colle-Salvetti correlation-energy formula into a functional of the electron density. Phys. Rev. B 1988, 37 (2), 785-789.

(3) Grimme, S.; Hujo, W.; Kirchner, B. Performance of dispersion-corrected density functional theory for the interactions in ionic liquids. Phys. Chem. Chem. Phys. 2012, 14 (14), 4875-4883.

(4) Izgorodina, E. I.; Seeger, Z. L.; Scarborough, D. L. A.; Tan, S. Y. S. Quantum Chemical Methods for the Prediction of Energetic, Physical, and Spectroscopic Properties of Ionic Liquids. Chem. Rev. 2017, 117 (10), 6696-6754.

(5) Perlt, E.; Ray, P.; Hansen, A.; Malberg, F.; Grimme, S.; Kirchner, B. Finding the best density functional approximation to describe interaction energies and structures of ionic liquids in molecular dynamics studies. J. Chem. Phys. 2018, 148 (19), 193835-1-193835-20.

(6) Low, K.; Tan, S. Y. S.; Izgorodina, E. I. An ab initio Study of the Structure and Energetics of Hydrogen Bonding in Ionic Liquids. Front. Chem. 2019, 7 (208), 1-16.

(7) Petersson, a.; Bennett, A.; Tensfeldt, T. G.; Al-Laham, M. A.; Shirley, W. A.; Mantzaris, J. A complete basis set model chemistry. I. The total energies of closed-shell atoms and hydrides of the first-row elements. J. Chem. Phys. 1988, 89 (4), 2193-2218.

(8) Petersson, G.; Al-Laham, M. A. A complete basis set model chemistry. II. Open-shell systems and the total energies of the first-row atoms. J. Chem. Phys. 1991, 94 (9), 6081-6090.

(9) Rackers, J. A.; Wang, Z.; Lu, C.; Laury, M. L.; Lagardere, L.; Schnieders, M. J.; Piquemal, J.-P.; Ren, P.; Ponder, J. W. Tinker 8: software tools for molecular design. J. Chem. Theory Comput. 2018, 14 (10), 5273-5289. 